\title{
Femtosecond laser microfabrication of subwavelength structures in photonics
}

\author{
Vladimir Mezentsev ${ }^{a}$, Jovana S. Petrovic $^{a}$, Mykhaylo Dubov $^{a}$, Ian Bennion ${ }^{a}$, \\ Jürgen Dreher ${ }^{b}$, Holger Schmitz ${ }^{a, b}$, and Rainer Grauer ${ }^{b}$ \\ ${ }^{a}$ Photonics Research Group, Aston University, Birmingham B47ET, United Kingdom; \\ ${ }^{b}$ Theoretische Physik I, Ruhr-Universität Bochum, D-44780 Bochum, Germany
}

\begin{abstract}
This paper describes experimental and numerical results of the plasma-assisted microfabrication of subwavelength structures by means of point-by point femtosecond laser inscription. It is shown that the spatio-temporal evolution of light and plasma patterns critically depend on input power. Subwavelength inscription corresponds to the supercritical propagation regimes when pulse power is several times self-focusing threshold. Experimental and numerical profiles show quantitative agreement.
\end{abstract}

\section{INTRODUCTION}

Femtosecond (fs) laser inscription is one of the enabling technologies for manufacturing of sophisticated photonic devices. It is quickly becoming one of the mainstream methods for microfabrication of the flexible 3D structures. Nonlinear propagation of the femtosecond laser pulses in gaseous and solid transparent dielectric media is a fundamental physical phenomenon in a wide range of important applications such as laser lidars, laser micromachining (ablation) and microfabrication etc. These applications require very high intensity of the laser field, typically $10^{12}-10^{15} \mathrm{TW} / \mathrm{cm}^{2}$. Such high intensity leads to significant ionization and creation of electron-hole plasma. The presence of plasma results into significant multiphoton and plasma absorption and plasma defocusing. Consequently, the propagation effects appear extremely complex and result from competitive counteraction of the above listed effects and Kerr effect, diffraction and dispersion. It turns out that the strongly nonlinear effects such self-focusing followed by the pulse splitting are essential to determine fine structure of the inscribed patterns. These phenomena feature extremely complex dynamics of both the electromagnetic field and plasma density with different spatio-temporal structures evolving at the same time.

The objective of this paper is to determine conditions for inscription of the smallest possible photonic structures routinely used e.g. in Bragg Gratings (BG). Typically, Bragg gratings are ubiquitous components in many composite devices where wavelength selectivity is ultimately required. Manufacturing of the BGs in bulk material is a challenging technological problem involving $10 \mathrm{~nm}$ accurate fabrication of periodic perturbation of refractive index within or in the vicinity of the waveguide. Femtosecond inscription is a unique technology enabling the fabrication of both the wave guide and the grating in the same set up. The major problem is a submicron pitch size for variation of refractive index required for the First Order BG (FOBG) due to diffraction limit. Photonic applications often imply grating period of $535 \mathrm{~nm}$ in fused silica to to satisfy the first order Bragg reflection condition for vacuum wavelength of $1550 \mathrm{~nm}$. Such application constraint requires pitch size of refractive index of about $260 \mathrm{~nm}$. In this paper we describe fs fabrication of the periodic structure of $25 \mathrm{~mm}$ long with such pitch in fused silica. This pitch size is less than a third of the wavelength of the laser light used (800 nm Ti:Sa laser). This is the smallest reported period for BG manufactured in the bulk of glass sample by means of point-by-point method. We also describe detailed numerical modelling of submicron inscription. It turns out that a submicron regime regime requires a supercritical self focusing to produce small scale plasma filaments. In this paper, we outline the role of plasma created during the course of propagation of the intense femtosecond (fs) laser pulse. To be more specific, the applications of this phenomenon in fs microfabrication used as an example. Direct inscription of the complex microstructures in refractive materials by means of intense femtosecond radiation is one of the novel enabling technologies in modern photonics. This technology implies that pre-focused femtosecond light

e-mail: v.mezentsev@aston.ac.uk

Laser-based Micro- and Nanopackaging and Assembly, edited by Wilhelm Pfleging, Yongfeng Lu, Kunihiko Washio, Friedrich G. Bachmann, Willem Hoving, Proc. of SPIE Vol. 6459, 64590B, (2007) · 0277-786X/07/\$18 - doi: 10.1117/12.705800

Proc. of SPIE Vol. 6459 64590B-1 
pulses produce phase transitions and create domains with a modified refractive index. Nonlinear propagation of femtosecond intense laser pulses in dielectrics exhibits a wide range of fascinating phenomena including conical emission, ${ }^{1}$ X-waves, ${ }^{2}$ self-reconstruction, ${ }^{3}$ self-healing ${ }^{2}$ light filaments plasma breakdown. ${ }^{4}$ Proof of principle experiments of the potential of fs inscription for microfabrication of photonic structures was demonstrated almost a decade ago. ${ }^{5}$ It has now become a very promising method of microfabrication. ${ }^{6-8}$ Other interesting applications aim to achieve the longest possible self-guiding filaments to deliver the energy through the bulk of material. ${ }^{9}$

Self-focusing of the intense laser pulse is a key physical phenomenon leading to a multi-photon ionization at its final stage. In fact the very formation of plasma filaments limits the catastrophic damage due to defocusing and multi-photon absorption. Eventually, the thermalization and recombination of the plasma filament leads to the modification of medium and a distributed profile of refractive index is produced. The dynamics of the light-induced plasma filaments is extremely complex and defined by many factors. It is an extremely fast process evolving at the very fine spatial scales.

\section{EXPERIMENTAL SETUP AND RESULTS}

The schematic of the experimental set-up is presented in Figure 1. It is similar to that used for the point-bypoint experiments ${ }^{10}$ but a fused silica slide was positioned horizontally to exploit the advantages of the $2 \mathrm{D}$ patterning in a transverse geometry. Pulse source comprises the laser system (Spectra Physics), the Spitfire amplifier (Positive Light), and Tsunami femtosecond mode-locked generator (Spectra Physics). It is capable to produce 120-150 fs pulses at the wavelength of $796 \mathrm{~nm}$ at $1 \mathrm{kHz}$ repetition rate. The computer controlled attenuation is realized with the half wave-plate (2) mounted on the motorized rotational stage, and a Glan prism (3). Electronic shutter (1) is installed immediately after the laser system output window. He-Ne laser (not shown in the Figure) is used for alignment.

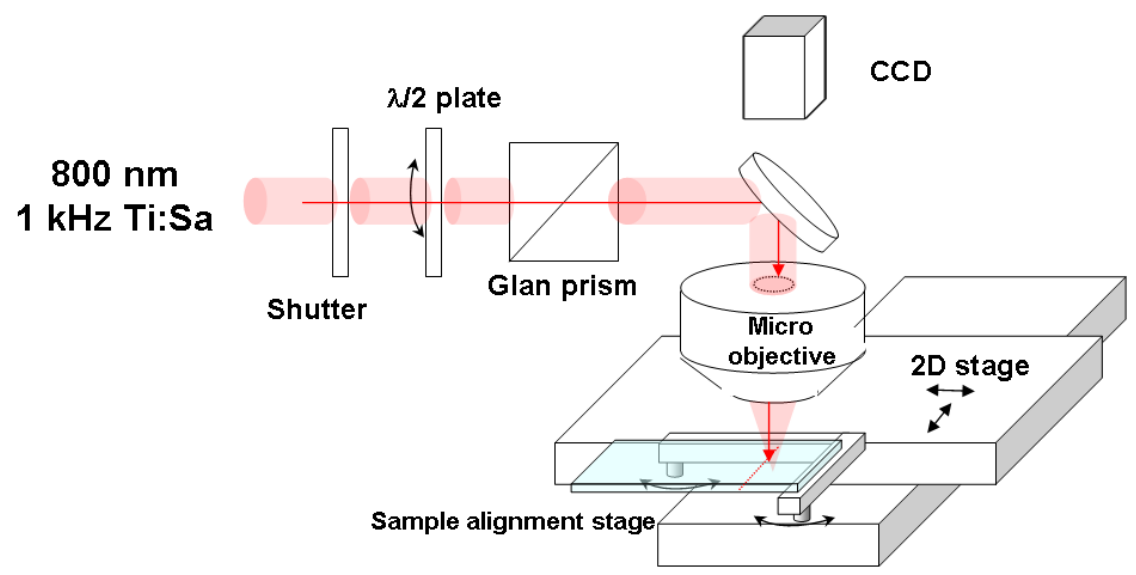

Figure 1. Experimental setup for implementation of plasma assisted material processing in the bulk of transparent dielectric. The light passes a shutter, a half-wave plate, Glan prism, and beamsplitter. It is focused by a $\times 36$ long working distance microscopic objective, into fused silica sample $50 \times 20 \times 1 \mathrm{~mm}$. The remote CCD camera was used for monitoring. The translation of the sample in a horizontal plane was performed by the $2 \mathrm{D}$ computer controlled stage.

The femtosecond inscription of all structures was performed after careful positioning of the sample surface at the same distance from the objective on both ends of the scanning range with the help of a modified mirror mount. We have used $\times 36 \mathrm{NA}=0.5$ long working distance Schwarzschield reflecting objective to focus the laser radiation. This objective has a variable correction ring (to correct a spherical aberration of a sharply focused beam) for the sample thickness - focusing depth - up to $1 \mathrm{~mm}$. The optimal correction was found as a result of multiple thorough experiments for smallest period of the grating. The remote CCD-camera was used for alignment and on-line monitoring of the inscription process. The z-axis stage (not shown on the Figure) was mounted on top of the stage. For grating fabrication we used mainly scanning in Y-direction, as this provides 

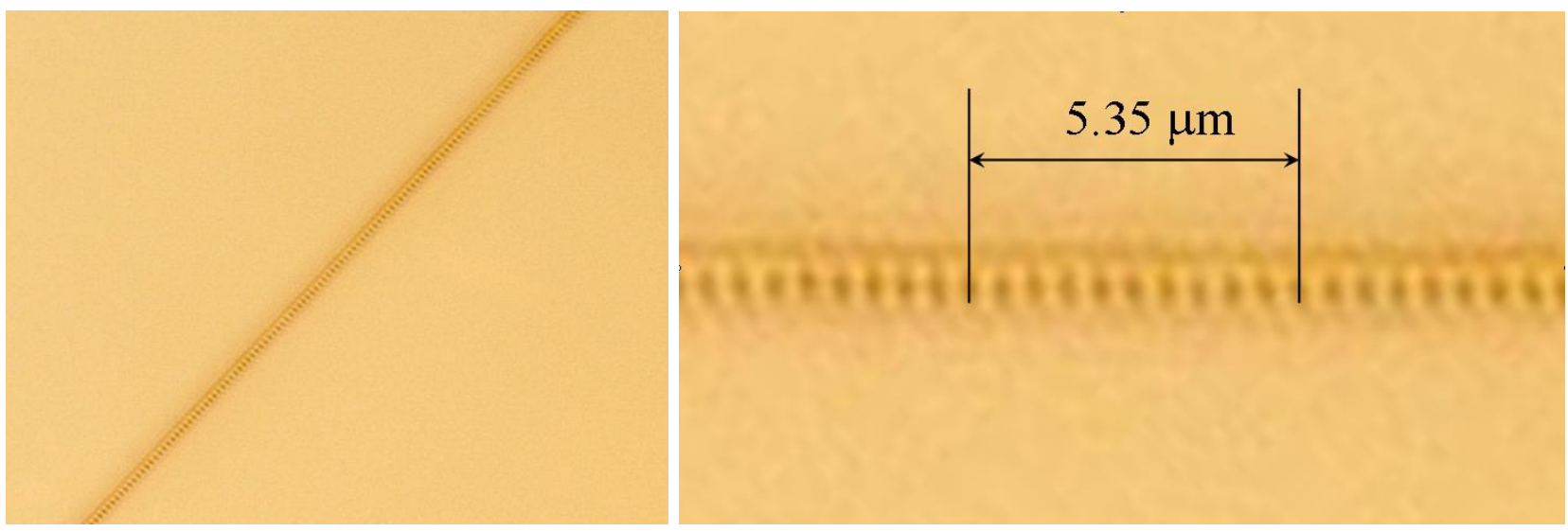

Figure 2. Grating closup.

for a triple reduction of the errors in positioning $(<50 \mathrm{~nm})$ as compared with the motion along the $\mathrm{X}$ (bottom axis). The objective has an obscuration about $15 \%$, and a working distance of $8.6 \mathrm{~mm}$. For inscription of the $535 \mathrm{~nm}$ period the stage was moved with a speed of $0.535 \mathrm{~mm} / \mathrm{sec}$ while the laser had a $1 \mathrm{kHz}$ repetition rate. Typical pulse energy for grating fabrication was $600 \mathrm{~nJ}$ which is approximately five times self-focusing power.

\section{THEORETICAL MODEL}

\subsection{Equations}

This section describes a theoretical model used for femtosecond pulse propagation in dielectrics. Electromagnetic wave is described by a set of Maxwell equations

$$
\begin{aligned}
& \nabla \times \mathbf{E}=-\frac{\partial \mathbf{B}}{\partial t} \\
& \nabla \times \mathbf{H}=\frac{\partial \mathbf{D}}{\partial t}+\mathbf{J} \\
& \mathbf{D}=\varepsilon \mathbf{E} ; \quad \mathbf{B}=\mu \mathbf{H},
\end{aligned}
$$

where $\mathbf{J}$ is an electron current density.

Description of plasma is based on relaxation dynamics of the electrons driven by the electromagnetic wave. The major source of plasma in strong electromagnetic field is multi-photon and avalanche ionization to be included in the continuity equation.

$$
\begin{aligned}
& \frac{d \mathbf{v}_{\mathbf{e}}}{d t}=-\tau_{c}^{-1} \mathbf{v}_{\mathbf{e}}-\frac{e}{m_{e}} e \mathbf{E} \\
& \mathbf{J}=-e \rho \mathbf{v}_{\mathbf{e}} \\
& \frac{d \rho}{d t}=\text { ionization sources }
\end{aligned}
$$

where $\tau_{c}$ is the shortest electron collision time. Envelope approximation can be used to describe quasi-monochromatic paraxial evolution

$$
\begin{aligned}
& \mathbf{E}\left(\mathbf{r}_{\perp}, z, t\right)=\hat{\mathbf{y}} \mathcal{E}\left(\mathbf{r}_{\perp}, z, t\right) \exp [i(k z-\omega t)] \\
& \frac{\partial}{\partial z}=i k+\frac{\partial}{\partial z} ; \quad \frac{\partial}{\partial t}=-i \omega+\frac{\partial}{\partial t}
\end{aligned}
$$


Finally, Kerr nonlinearity must be taken into account for strong laser field $n=\sqrt{\varepsilon}=n_{0}+n_{2}|\mathcal{E}|^{2}$. Such approach was originally suggested by Feit and Fleck ${ }^{11}$ and later developed into fairly complex models, see e.g. Refs. ${ }^{9,12-18}$ For the purposes of this paper, a simplified model is used, essentially similar to that described by Feng et al. ${ }^{19}$ :

$$
\begin{gathered}
i \mathcal{E}_{z}+\frac{1}{2 k} \Delta_{\perp} \mathcal{E}-\frac{k^{\prime \prime}}{2} \frac{\partial^{2} \mathcal{E}}{\partial t^{2}}+k_{0} n_{2}|\mathcal{E}|^{2} \mathcal{E}=-\frac{i \sigma}{2}(1+i \omega \tau) \rho \mathcal{E}-i \frac{\beta^{(K)}}{2}|\mathcal{E}|^{2(K-1)} \mathcal{E} \\
\frac{\partial \rho}{\partial t}=\frac{1}{n_{b}^{2}} \frac{\sigma_{b s}}{E_{g}} \rho|\mathcal{E}|^{2}+\frac{\beta^{(K)}}{K \hbar \omega}|\mathcal{E}|^{2 K}
\end{gathered}
$$

The terms on the left-hand side of Eq.(1) describe effects of beam diffraction, group velocity dispersion (GVD), and Kerr nonlinearity. The latter is responsible for a catastrophic self-focusing which is limited by the effects described by terms on the right-hand side of Eq.(1), namely plasma absorption and multi-photon absorption. In Eq.(1) the laser beam propagation along the $z$ axis is assumed and this equation is essentially a reduced paraxial approximation of the wave equation for the complex electric field envelope $\mathcal{E}$ with a carrier frequency $\omega$ in the moving frame of coordinates. Here $k=n_{b} k_{0}=n_{b} \omega / c$ is the propagation vector, $k^{\prime \prime}=\partial^{2} k(\omega) / \partial \omega^{2}$ is the GVD parameter, $n_{b}(\omega)$ is a linear refractive index of the bulk medium, $n_{2}$ is the nonlinear coefficient describing nonlinear self-modulation (Kerr effect) such that $n_{2}|\mathcal{E}|^{2}$ is a nonlinear contribution to the refractive index, $\sigma_{b s}$ is the cross section for inverse Bremsstrahlung, $\tau$ is the electron relaxation time, $E_{g}$ is the ionization energy, and the quantity $\beta^{(K)}$ controls the $K$-photon absorption. Equation (2) implements the Drude model for electron-hole plasma in the bulk of silica and describes the evolution of the electron density $\rho$. The first term on the right-hand side is responsible for the avalanche impact ionization and the second term - for the ionization resulting from MPA. Equation (2) is suitable for description of the sub-picosecond laser pulses when plasma diffusion is negligible. Here, the wave equation describing the evolution of the focused optical beam in the form of NLSE (left-hand terms in Eq.(1)) which is extended to include plasma generation, pulse-plasma interaction, and MPA (terms on the right-hand of Eq.(1). Group velocity dispersion included in Eq.(1) has been shown to lead to pulse splitting and to arrest the collapse. ${ }^{20-25}$

\subsection{Plasma parameters}

This section describes a set of typical parameters for plasma generated by the femtosecond pulse. We consider a couple of limiting factors which define the range of plasma concentration and temperature. One important limitation can be defined from the matching condition between the laser frequency $f_{e}$ and plasma frequency $f_{p}=\omega_{p} / 2 \pi=(2 \pi)^{-1}\left(\rho e^{2} / \varepsilon_{0} m_{e}\right)^{1 / 2}$ where $\rho$ is an electron concentration. The condition $f_{e}=f_{p}$ defines the critical (breakdown) density of plasma $\rho_{B D}=\varepsilon_{0} m_{e} e^{-2} \omega^{2}$. This is a resonant condition for conversion of electromagnetic wave into plasma waves. It defines a breakdown electron density $\rho_{B D}$ used below for a given laser frequency.

The plasma temperature can be estimated from the analysis of the electron oscillations in high frequency electromagnetic field. Then average kinetic energy can be expressed as

$$
K=\left\langle\frac{e^{2} \mathcal{E}(t)^{2}}{4 m_{e} \omega^{2}}\right\rangle_{t}=9.3 \times 10^{-14} I \lambda^{2},
$$

where $e$ and $m_{e}$ are electron charge and mass respectively, $\mathcal{E}$ is an envelope amplitude of the electric field and $I$ is its intensity, omega is a carrier angular frequency of the electromagnetic wave, and $\lambda$ is its wavelength. The numerical expression in the right hand side gives a value of the kinetic value in $\mathrm{eV}$ provided that intensity is given in $\mathrm{W} / \mathrm{cm}^{2}$ and the wavelength is given in microns. Typical values of electron temperatures versus laser intensity are summarized in Table 1.

\begin{tabular}{lccc}
\hline Intensity, $\mathrm{W} / \mathrm{cm}^{2}$ & $10^{13}$ & $10^{14}$ & $10^{15}$ \\
Electron energy, eV & 0.01 & 1 & 100 \\
\hline
\end{tabular}

Table 1. Average electron energy versus light intensity for typical focusing conditions 


\subsection{Physical parameters}

In all our simulations the Gaussian initial condition:

$$
\mathcal{E}(z=0, r, t)=\sqrt{\frac{2 P_{i n}}{\pi r_{0}^{2}}} \exp \left(-\frac{r^{2}}{r_{0}^{2}}-\frac{i k r^{2}}{2 f}-\frac{t^{2}}{t_{p}^{2}}\right),
$$

where $r_{0}$ is the waist of the incident beam, $t_{p}$ defines the conventionally defined pulsewidth $t_{F W H M}=\sqrt{2 \ln 2} t_{p} \approx$ $1.177 t_{p}$, and $f$ is a focal length of the objective lens.

For all our simulations a fixed single values of $r_{0}=2.5 \mathrm{~mm}$ and $t_{p}=60 \mathrm{fs}$ was used. This fixed pulsewidth corresponds to the critical energy of $116 \mathrm{~nJ}$ for a critical power $P_{c r}=\lambda_{0}^{2} / 2 \pi n_{b} n_{2} \approx 2.3 \mathrm{MW}$ in fused silica with $n_{b}=1.453$ being the linear refraction index and $n_{2}=3.2 \times 10^{-16} \mathrm{~cm}^{2} / \mathrm{W}$ the nonlinear refraction index. Critical power is proven to be a crucial parameter to determine the evolution of the collapsing beam. We assume the laser wavelength $\lambda_{0}$ to be $800 \mathrm{~nm}$ and the focusing lens with $f$ corresponding to $\mathrm{NA}=0.4$.

The other parameters for fused silica, used in simulations are described below. GVD coefficient $k^{\prime \prime}=361$ $\mathrm{fs}^{2} / \mathrm{cm}$, inverse Bremsstrahlung cross section $\sigma_{b s}=2.78 \times 10^{-18} \mathrm{~cm}^{2}$. Multiphoton absorption coefficient can be expressed as $\beta^{(K)} \hbar \omega \sigma_{K} \rho_{a t}$, whith $\rho_{a t}=2.1 \times 10^{22}$ atoms $/ \mathrm{cm}^{3}$ being a material concentration and $\sigma_{K}=$ $1.3 \times 10-55 \mathrm{~cm}^{2 K} / \mathrm{W}^{K} / \mathrm{s}$. We assume five-photon ionization with $K=5$ and $E_{g}=7.6 \mathrm{eV}$ in fused silica.

The Equation (2) can be expressed as

$$
\frac{\partial}{\partial t} \frac{\rho}{\rho_{B D}}=\frac{1}{n_{b}^{2}} \frac{\sigma_{b s}}{E_{g}} \frac{\rho}{\rho_{B D}}|\mathcal{E}|^{2}+\left(\frac{|\mathcal{E}|^{2}}{I_{M P A}}\right)^{K}
$$

where

$$
I_{M P A}=\left(\frac{K \hbar \omega \rho_{B D}}{\beta^{(K)}}\right)^{1 / K}
$$

is defined to be an MPA threshold as the plasma ionization rate becomes very steep when the intensity $I$ exceeds $I_{M P A} \cdot \rho_{B D}=1.7 \times 10^{21}$ is a plasma breakdown density. Being important physical thresholds, both $I_{M P A}$ and $\rho_{B D}$ were used in a very useful normalization of physical variables to dimensionless ones for performing simulations. The choice of normalization is irrelevant to discuss as all the parameters and fields throughout this paper are produced with their physical values.

\section{NUMERICAL MODELING}

Equations Eqs $(1,2)$ were used for numerical modeling of the plasma formation using adaptive mesh approach described earlier. ${ }^{26}$ We use a simplified version of the widely accepted model of the nonlinear propagation of the laser pulse as it was formulated by Feng et al Ref.. ${ }^{19}$ It is essentially a nonlinear Schrödinger equation (NLSE) coupled with an equation describing the plasma generation. This basic model includes effects of self-focusing, multiphoton absorption (MPA), and group velocity dispersion (GVD). Pure NLSE is a generic model which ultimately appears in consistent description of envelope amplitude of the nonlinear wave packets. It is widely used to describe fs light propagation ${ }^{27}$ and has been extensively studied. One of the most striking features of the NLSE is catastrophic self-focusing or beam collapse which means a formation of a singularity in finite propagation length. ${ }^{28}$ Beam collapse happens in the framework stationary NLSE if the pulse power exceeds a certain critical value. Formally, the on-axis intensity achieves an infinitely high value at some critical propagation length. However, in the extended physical models which account for dispersion and nonlinear absorption a formation of singularity is arrested. ${ }^{25}$ The nonlinear evolution of the collapsing beam with the presence of the arresting effects is extremely complex..$^{25,26}$ Mathematically it poses a stiff multidimensional evolution problem. Our algorithm to resolve an extremely stiff propagation dynamics is very similar to the one used by Berger and Colella, ${ }^{29}$ and it was described in detail by Friedel et al.. ${ }^{30}$ Application of this method to the Eqs $(1,2)$ was described in our earlier work ${ }^{25,26}$ 

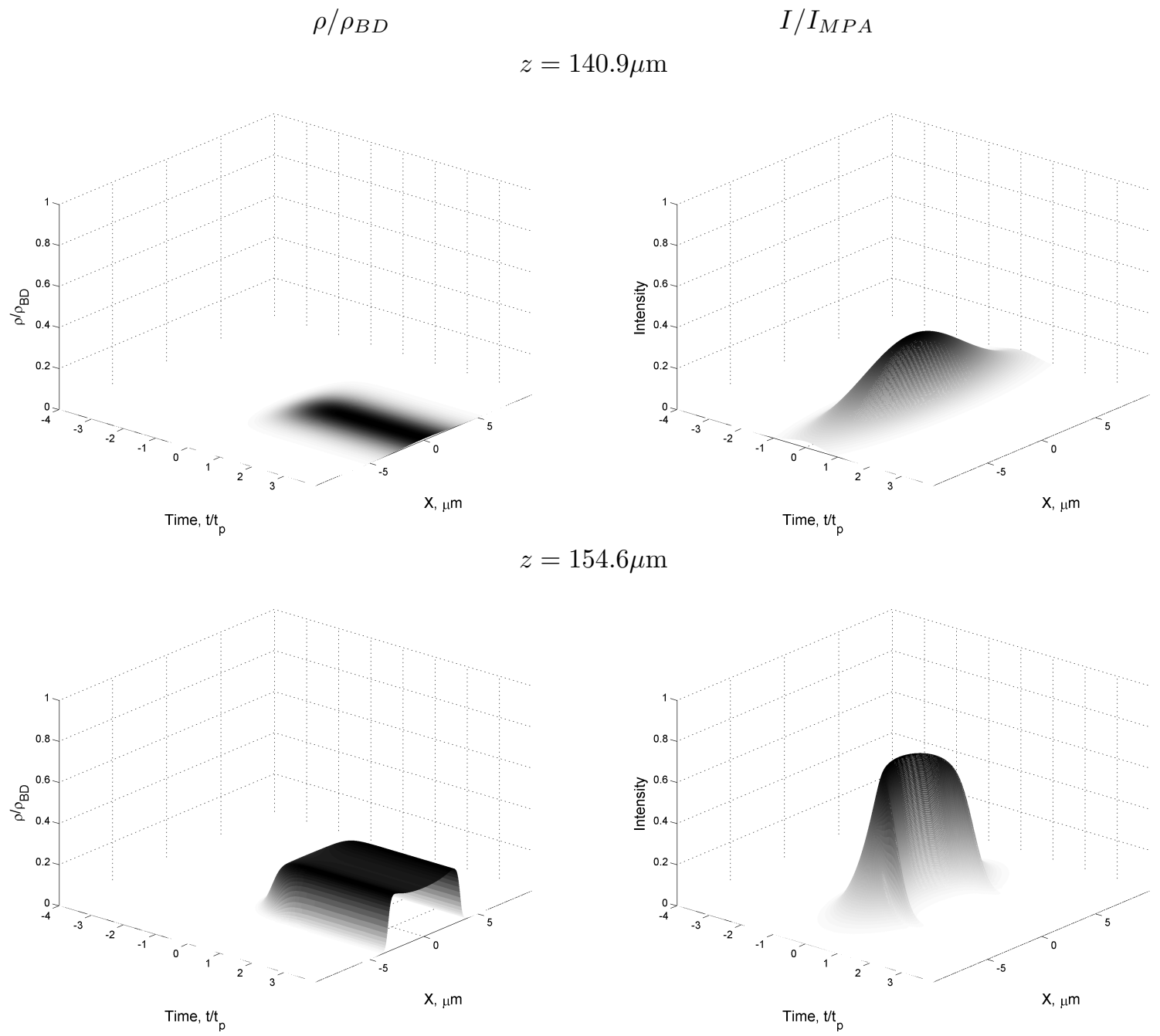

$z=165.9 \mu \mathrm{m}$
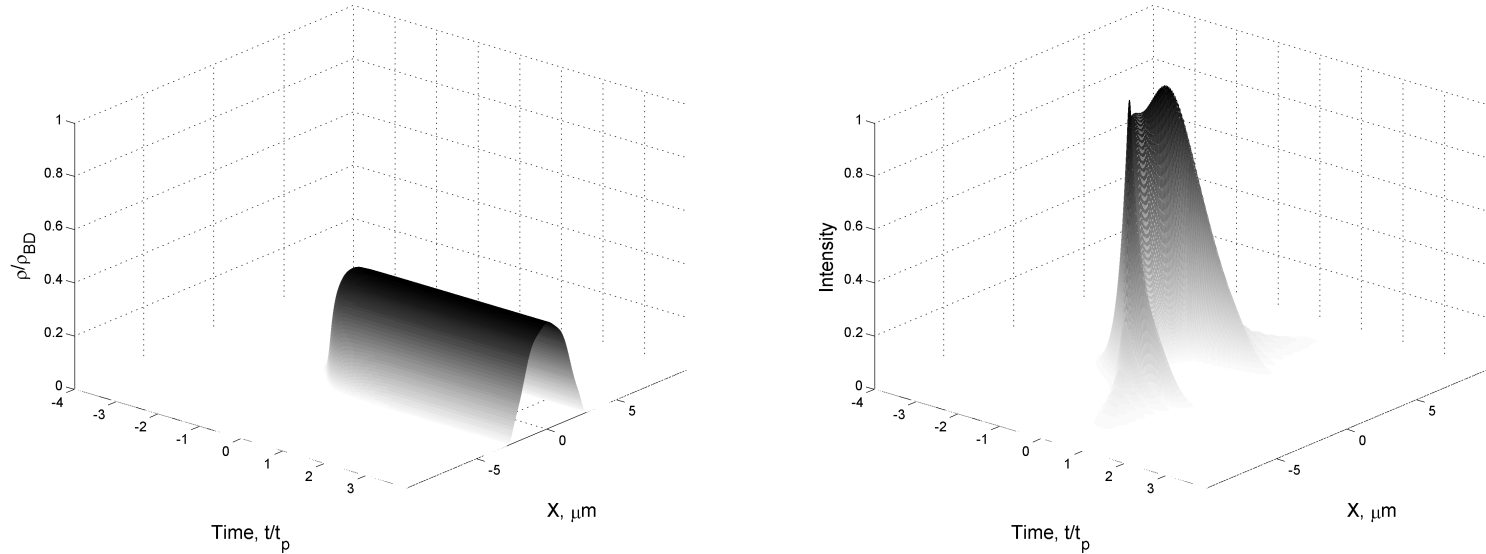

Proc. of SPIE Vol. 6459 64590B-6 

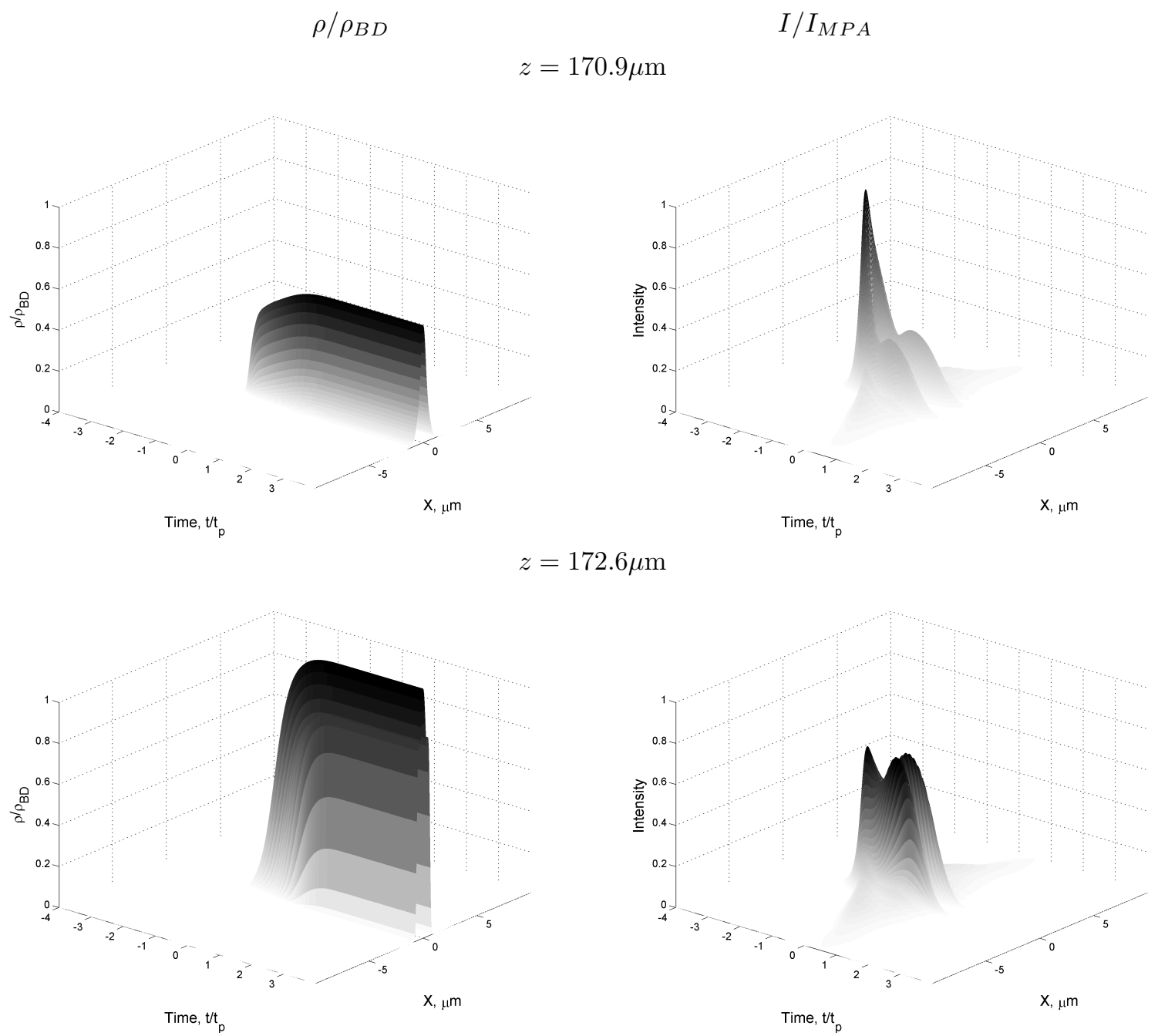

$z=175.4 \mu \mathrm{m}$
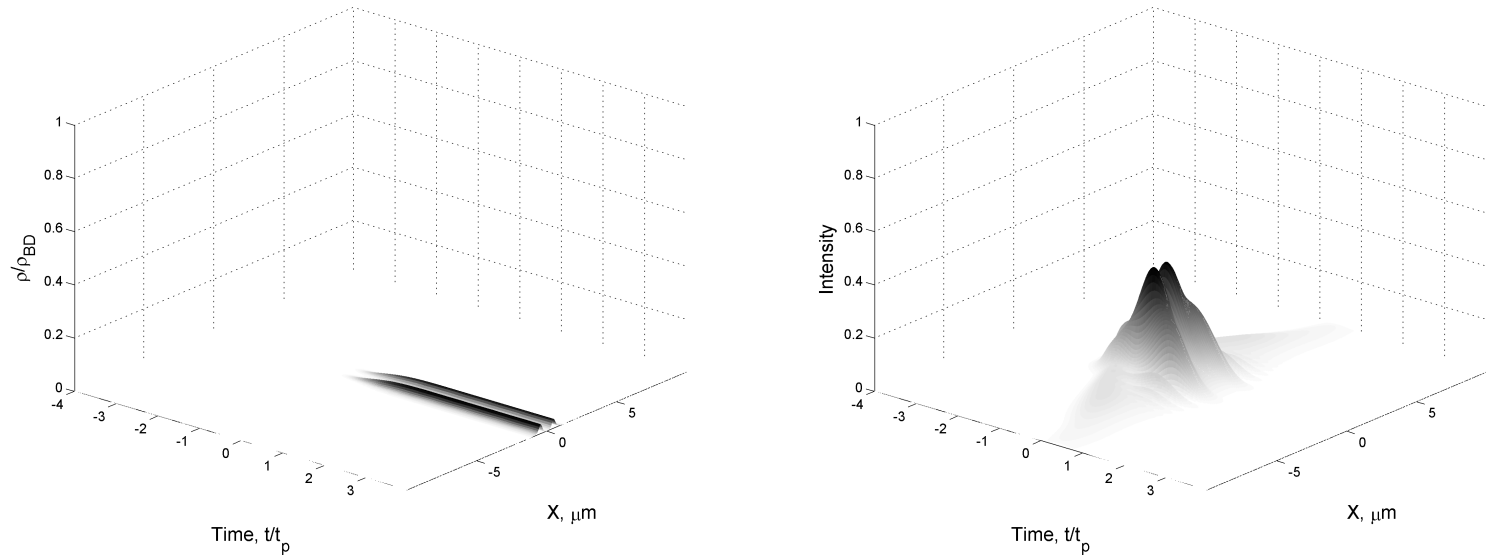

Figure 3. Dynamics of the intensity and the plasma density at different positions along axis $z$. 

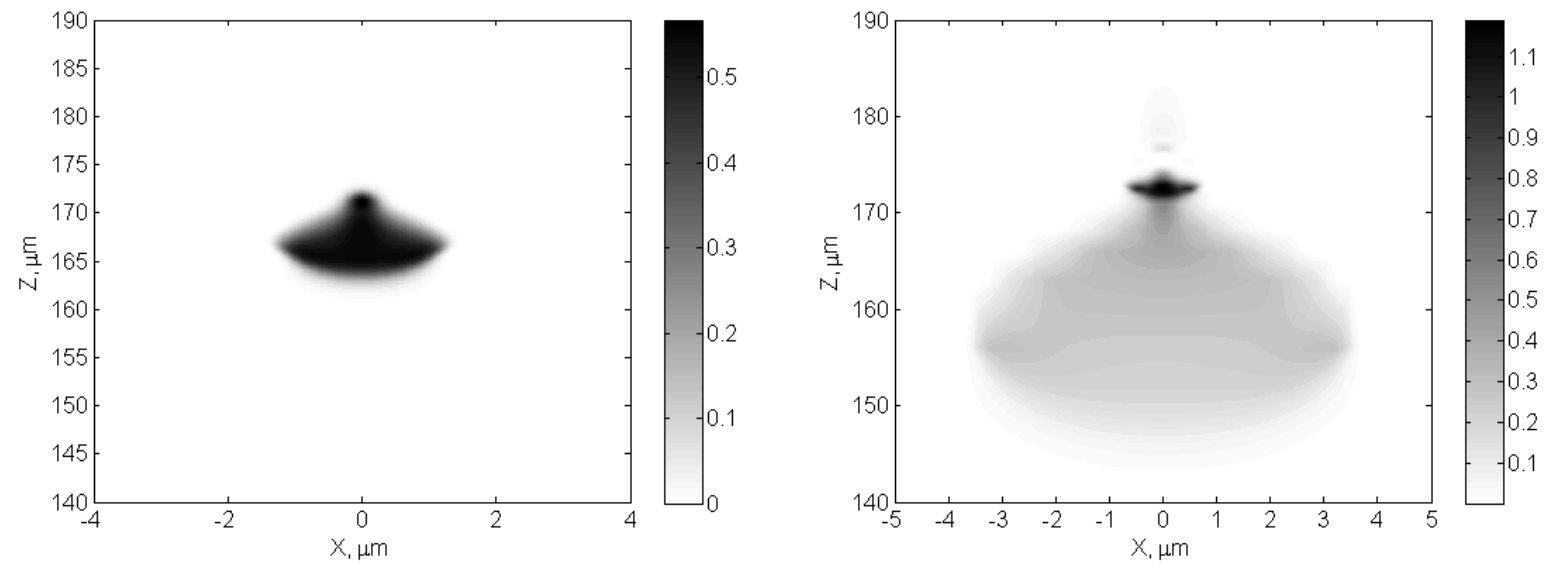

Figure 4. Contours of the plasma density at infinite time after electric field is vanished for different input powers: subcritical $P_{\text {in }}=0.5 P_{c r}$ (left) and supercritical $P_{\text {in }}=5 P_{c r}$ (right).
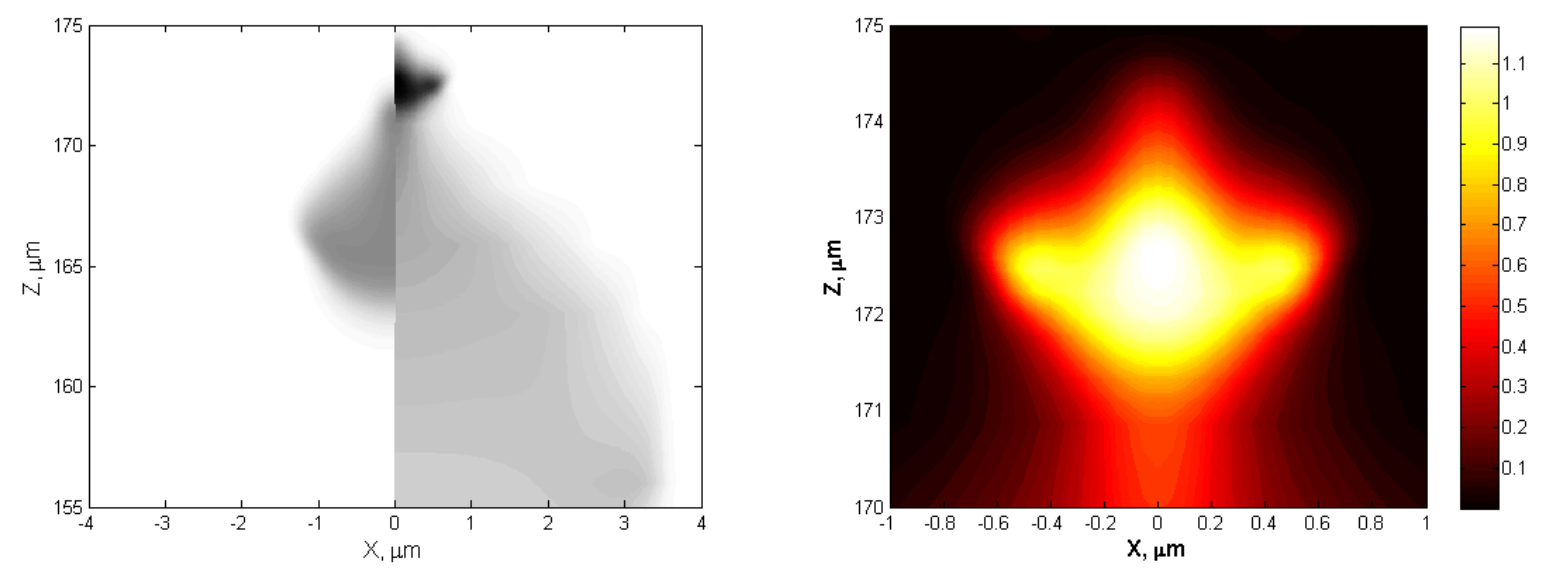

Figure 5. Left: Back-to-back comparison of the plasma produced in subcritical and super-critical regimes. Right: Closeup of the plasma profile in super-critical case.

\section{NUMERICAL RESULTS AND INTERPRETATION}

There are two distinct typical setups related to the focusing geometry. If the goal is to produce the longest possible filament, then the loose focusing and small spot size are required. ${ }^{2,9,31}$ However in the microfabrication context the opposite goal is usually desirable. The focused spot is often required to be as small as possible and the absorbed energy is needed to be within a narrow window between the thresholds of inscription and damage. This is an extremely difficult challenge because of the huge difference in spatial and temporal scales of the incident laser beam and fine features of light and plasma patterns in the vicinity of the focal point. The adaptive procedure $^{26}$ allows for accurate treatment of multiscale evolution which results in stationary (in the framework of the model considered) distribution of plasma. The mechanisms of eventual plasma recombination and subsequent relaxation of the medium are extremely complex. For example, the latter can be described as a sophisticated 3D thermo-elasto-plastic processes. ${ }^{32}$

Nontrivial complex light and plasma dynamics and formation of interesting light patterns are illustrated by Figure 3. Snapshots of the beam intensity profile in transverse space and time are presented at different points 

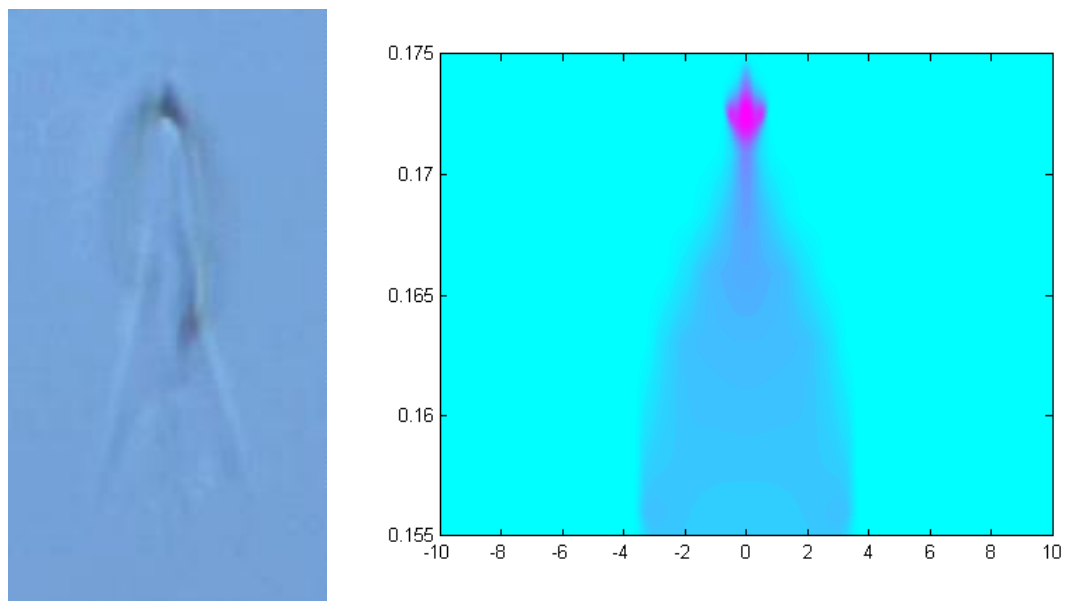

Figure 6. Comparison of the microscopic image of the inscribed microsstructure (left) and plasma profile (right) obtained numerically for comparable conditions.

along the propagation axis in the vicinity of the focal point for supercritical case $P_{\text {in }} \approx 5 P_{c r}$ corresponding to the experimentally found inscription regime. Originally, an intensity profile forms a distinct crescent in $x-t$ plane. It is then evolved so that the shoulders of this crescent split from the front of the pulse and form a pair of satellite pulses which rise being fed by a contracting beam.

The purpose of the adopted approach is to find an accurate spatial distribution of plasma needed for such or similar subsequent analysis. Hence the most important result of the intense laser propagation is creation of the cloud of plasma due to ionization. The stationary spatial distribution of electrons can be found as

$$
\rho(\mathbf{r}, z)=\int_{-\infty}^{\infty}\left[\frac{1}{n_{b}^{2}} \frac{\sigma}{E_{g}} \rho|\mathcal{E}(\mathbf{r}, z, t)|^{2}+\frac{\beta^{(K)}}{K \hbar \omega}|\mathcal{E}(\mathbf{r}, z, t)|^{2 K}\right] d t
$$

Typical stationary plasma profiles for different initial pulse energies are shown in Fig. 4. Density plots of plasma concentrations are shown in one transverse and one propagation coordinates. It shows that subcritical evolution $\left(P_{i n}<P_{c r}\right)$ leads to a smooth relatively large plasma cloud without visible fine structure. This regime is probably the most attractive for microfabrication of large scale structures such as waveguides because it features smooth subcritical evolution of the peak intensity. Larger energies lead to development of the pronounced small scale core developed after initial nonlinear pre-focusing. Collapse of the laser beam due to self-focusing is eventually arrested by multiphoton absorption resulting in a very confined plasma cloud. Figure 5 shows back-to-back comparison of the plasma profiles resulting from the subcritical $\left(P_{i n}=0.5 P_{c r}\right)$ and super-critical $\left(P_{i n}=5 P_{c r}\right)$ focusing regimes. Figure 5 (right plot) shows a closeup of the domain with dense plasma created as a result of the supercritical self-focusing. It is seen that the transverse size of the plasma is about $400 \mathrm{~nm}$. This distribution is assumed to be translate into the distribution of the material temperature since the energy density of the thermalized plasma is proportional to the plasma density. Extrapolation of the results of the numerical modeling of the irreversible thermo-elastic dynamics ${ }^{32}$ suggests that the resulting diameter of the domain with modified refractive index will be a half of that for the temperature profile. This suggest the estimate of the pitch size of refractive index corresponding to the regime $P_{i n}=5 P_{c r}$ to be about $200 \mathrm{~nm}$ which is in excellent agreement with the observed structure. A comparison of the microscopic image of this structure with the numerically found plasma profile is shown in Fig 6

\section{CONCLUSION}

It is shown that femtosecond inscription of the subwavelength photonic structures is feasible for the supercritical self-focusing regimes. Experimentally found regime was numerically modelled by means of adaptive mesh code to reveal a nonlinear propagation regime leading to creation of the confined plasma cloud. Recombination of this 
plasma is suggested to be an energy source for eventual densification which manifests in subwavelength pitches of refractive index.

\section{ACKNOWLEDGMENTS}

We acknowledge support of the technical staff running Cray XD1 computing cluster facility in School of Engineering and Applied Science at Aston University. This work was funded by EPSRC Grant EP/D060990/1.

\section{REFERENCES}

1. O. G. Kosareva, V. P. Kandidov, A. Brodeur, C. Y. Chien, and S. L. Chin, "Conical emission from laserplasma interactions in the filamentation of powerful ultrashort laser pulses in air," Opt. Lett. 22, pp. 13321334, 1997.

2. M. Kolesik, E. M. Wright, and J. Moloney, "Dynamic nonlinear $\mathrm{x}$-waves for femtosecond pulse propagation inwater," Phys. Rev. Lett. 92, p. 253901, 2004.

3. A. Dubietis, E. Kucinskas, G. Tamosauskas, E. Gaizauskas, M. A. Porras, and P. D. Trapani, "Selfreconstruction of light filaments," Opt. Lett. 29, pp. 2893-2895, 2004.

4. C. W. Carr, M. D. Feit, A. M. Rubenchik, P. D. Mange, S. O. Kucheyev, M. D. Shirk, H. B. Radousky, and S. G. Demos, "Radiation produced by femtosecond laserplasma interaction during dielectric breakdown," Opt. Lett. 30, pp. 661-663, 2005.

5. K. M. Davis, K. Miura, N. Sugimoto, and K. Hirao, "Writing waveguides in glass with a femtosecond laser," Opt. Letters 21, pp. 1729-1731, 1996.

6. C. B. Schaffer, A. Brodeur, J. F. Garcìa, and E. Mazur, "Micromachining bulk glass by use of femtosecond laser pulses with nanojoule energy," Opt. Lett. 26, pp. 93-95, 2001.

7. A. H. Nejadmalayeri, P. R. Herman, J. Burghoff, M. Will, S. Nolte, and A. Tuennermann, "Inscription of optical waveguides in crystalline silicon by mid-infrared femtosecond laser pulses," Opt. Lett. 30, pp. 964966, 2005.

8. A. M. Kowalevicz, V. Sharma, E. P. Ippen, J. G. Fujimoto, and K. Minoshima, "Three-dimensional photonic devices fabricated in glass by use of a femtosecond laser oscillator," Opt. Lett. 30, pp. 1060-1062, 2005.

9. S. Tzortzakis, L. Sudrie, M. Franco, B. Prade, A. Mysyrowicz, A. Couairon, and L. Bergé, "Self-guided propagation of ultrashort ir laser pulses in fused silica," Phys. Rev. Lett. 21, p. 213902, 2001.

10. V. Mezentsev, M. Dubov, A. Martinez, Y. Lai, T. P. Allsop, I. Khrushchev, D. J. Webb, F. Floreani, and I. Bennion, "Micro-fabrication of advanced photonic devices by means of direct point-by-point femtosecond inscription in silica," in Laser-based Micropackaging, F. G. Bachmann, W. Hoving, Y. Lu, and K. Washio, eds., Proc. SPIE 6107, p. 61070C, 2006.

11. M. D. Feit and J. A. Fleck, "Effect of refraction on spot-size dependence of laser-induced breakdown," Appl. Phys. Lett. 24, pp. 169-172, 1974.

12. T. Brabec and F. Krausz, "Nonlinear optical pulse propagation in the single-cycle regime," Phys. Rev. Lett. 78, pp. 3282-3285, 1997.

13. J. K. Ranka and A. L. Gaeta, "Breakdown of the slowly varying envelope approximation in the self-focusing of ultrashort pulses," Opt. Lett. 23, pp. 534-536, 1998.

14. A. L. Gaeta, "Catastrophic collapse of ultrashort pulses," Phys. Rev. Lett. 84, pp. 3582-3585, 2000.

15. M. Kolesik, J. V. Moloney, and M. Mlejnek, "Unidirectional optical pulse propagation equation," Phys. Rev. Lett. 89, p. 283902, 2002.

16. S. Tzortzakis, L. Bergé, M. Franco, B. Prade, A. Mysyrowicz, and A. Couairon, "Infrared femtosecond light filaments in air: simulations and experiments," J. Opt. Soc. Am. B 19, pp. 1117-1129, 2002.

17. M. Kolesik, G. Katona, J. Moloney, and E.M.Wright, "Physical factors limiting the spectral extent and band gap dependence of supercontinuum generation," Phys. Rev. Lett. 91, p. 043905, 2003.

18. R. Nuter, S. Skupin, and L. Bergé, "Chirp-induced dynamics of femtosecond filaments in air," Opt. Lett 30, pp. 917-919, 2005. 
19. Q. Feng, J. V. Moloney, A. C. Newell, E. M. Wright, K. Cook, P. K. Kennedy, D. X. Hammer, B. A. Rockwell, and C. R. Thompson, "Theory and simulation on the threshold of water breakdown induced by focused ultrashort laser pulses," IEEE Journal of Quantum Electronics 33, pp. 127-137, 1997.

20. N. A. Zharova, A. G. Litvak, T. A. Petrova, A. M. Sergeev, and A. D. Yunakovskii, "Multiple fractionation of wave structures in a nonlinear medium," Pisma Zh. Eksp. Teor. Fiz. (JETP Lett.) 44, pp. 12-15, 1986.

21. P. Chernev and V. Petrov, "Self-focusing of light pulses in the presence of normal group velocity dispersion," Opt. Lett. 17, pp. 172-174, 1992.

22. J. E. Rothenberg, "Pulse splitting during self-focusing in normally dispersive media," Opt. Lett. 17, pp. 583$585,1992$.

23. G. G. Luther, J. V. Moloney, A. C. Newell, and E. M. Wright, "Selffocusing threshold in normally dispersive media," Opt. Lett. 19, pp. 862-864, 1994.

24. G. G. Luther, A. C. Newell, and J. V. Moloney, "The effect of normal dispersion on collapse events," Physica D 74, pp. 59-73, 1994.

25. K. Germaschewski, R. Grauer, L. Bergé, V. K. Mezentsev, and J. J. Rasmussen, "Splittings, coalescence, bunch and snake patterns in the $3 \mathrm{~d}$ nonlinear schrodinger equation with anisotropic dispersion," Physica D 151, pp. 175-198, 2001.

26. V. Mezentsev, J. Petrovic, J. Dreher, and R. Grauer, "Adaptive modeling of the femtosecond inscription in silica," in Laser-based Micropackaging, F. G. Bachmann, W. Hoving, Y. Lu, and K. Washio, eds., Proc. SPIE 6107, p. 61070R, 2006.

27. J. H. Marburger, "Self-focusing: theory," Prog. Quantum Electron. 4, pp. 35-110, 1975.

28. S. Vlasov, V. Petrishchev, and V. Talanov Izv. Vuzov, Radiofizica 14, pp. 1353-1364, 1971.

29. M. J. Berger and P. Colella, "Local adaptive mesh refinement for shock hydrodynamics," J. Comp. Phys. 82, pp. 64-84, 1989.

30. H. Friedel, R. Grauer, and C. Marliani, "Adaptive mesh refinement for singular current sheets in incompressible magnetohydrodynamic flows," J. Comp. Phys. 134, pp. 190-198, 1997.

31. A. Dubietis, G. Tamoauskas, I. Diomin, and A. Varanaviiuset, "Self-guided propagation of femtosecond light pulses in water," Opt. Lett 28, pp. 1269-1271, 2003.

32. X. Zhang, X. Xu, and A. Rubenchik, "Simulation of microscale densification during femtosecond laser processing of dielectric materials," Appl. Phys. A 79, pp. 945-948, 2004. 